\title{
Role of the Absorbing Area in Chaotic Synchronization
}

\author{
Maistrenko, Yu.L.; Maistrenko, V.L.; Popovich, A.; Mosekilde, Erik
}

Published in:

Physical Review Letters

Link to article, DOI:

10.1103/PhysRevLett.80.1638

Publication date:

1998

Document Version

Publisher's PDF, also known as Version of record

Link back to DTU Orbit

Citation (APA):

Maistrenko, Y. L., Maistrenko, V. L., Popovich, A., \& Mosekilde, E. (1998). Role of the Absorbing Area in Chaotic Synchronization. Physical Review Letters, 80(8), 1638-1641. https://doi.org/10.1103/PhysRevLett.80.1638

\section{General rights}

Copyright and moral rights for the publications made accessible in the public portal are retained by the authors and/or other copyright owners and it is a condition of accessing publications that users recognise and abide by the legal requirements associated with these rights.

- Users may download and print one copy of any publication from the public portal for the purpose of private study or research.

- You may not further distribute the material or use it for any profit-making activity or commercial gain

- You may freely distribute the URL identifying the publication in the public portal

If you believe that this document breaches copyright please contact us providing details, and we will remove access to the work immediately and investigate your claim 


\title{
Role of the Absorbing Area in Chaotic Synchronization
}

\author{
Yu. L. Maistrenko, ${ }^{1}$ V. L. Maistrenko, ${ }^{1}$ A. Popovich, ${ }^{1}$ and E. Mosekilde ${ }^{2}$ \\ ${ }^{1}$ Institute of Mathematics, National Academy of Sciences, Kiev, Ukraine 252601 \\ ${ }^{2}$ Department of Physics, Technical University of Denmark, 2800 Lyngby, Denmark
}

(Received 14 July 1997)

\begin{abstract}
When two identical chaotic oscillators interact, one or more intervals of coupling parameters generally exist in which the synchronized state is weakly stable and its basin of attraction is riddled with holes that are repelled from it. Here, we discuss the role of the absorbing area for the emergence of local vs global riddling and for controlling the dynamics, once the synchronization breaks down. [S0031-9007(98)05306-X]
\end{abstract}

PACS numbers: 05.45.+b

Fujisaka and Yamada [1] have shown how two identical chaotic oscillators under variation of the coupling parameter can attain a state of chaotic synchronization in which the motion of the coupled system is restricted to an invariant subspace of total phase space. For two coupled onedimensional maps, for instance, the synchronized motion is one dimensional and occurs along the main diagonal in phase space. A variety of applications of this phenomenon are presently being investigated, particularly in connection with electronic communication and monitoring of dynamical systems [2].

An interesting problem concerns the starting conditions for which synchronization can be achieved. Recent studies of chaotic dynamics restricted to low-dimensional manifolds have led to the discovery of a variety of new phenomena, including on-off intermittency [3] and riddled basins of attraction $[4,5]$.

If the transverse Lyapunov exponent $\lambda_{\perp}$ is negative, the synchronous state, on the average, attracts points from its two-dimensional neighborhood [4]. In general, however, there will be intervals of the coupling parameter for which $\lambda_{\perp}<0$ while, at the same time, certain orbits embedded in the chaotic state are transversely unstable. The basin of attraction may then be riddled with tongues in which the trajectories are repelled from the low-dimensional manifold [6,7]. The synchronized state will attract a set of points of positive Lebesgue measure. Arbitrarily close to any such point, however, one can find a positive measure set of points that are repelled from this state, and the basin of attraction is locally a fat fractal.

For the synchronized state to be an attractor in the topological sense, all orbits on the chaotic set must be transversely stable. The riddling bifurcation (also referred to as the bubbling transition [5]) in which the first orbit embedded in the chaotic attractor loses its transverse stability has recently been investigated in detail by Lai et al. [8]. They suggest that the bifurcation takes place as two repellers located symmetrically on either side of the invariant subspace approach the chaotic attractor and collide with a saddle embedded in this attractor. Alternatively, a point cycle embedded in the synchronized state may lose its transverse stability in a period-doubling bifurcation [9], and both types of local bifurcation may be either soft or hard.

However, destabilization of orbits embedded in the chaotic state is not sufficient for directly observable riddling to occur. This will depend on the global dynamics of the system. Having left the locally repelling regions in the neighborhood of the invariant manifold, the trajectories may wander around in phase space without ever approaching another attractor (or escaping to infinity). Sooner or later, most of them will return to the neighborhood of the invariant manifold. Some may again be mapped into the repelling tongues while others will be attracted by the manifold. This gives rise to a dynamics with temporal bursting (attractor bubbling) and very long transients. However, at the end, almost all initial conditions close to the invariant manifold will end up in the synchronized state.

Denoting the synchronized chaotic state by $A$, its $\delta$ neighborhood by $U_{\delta}(A)$, and its basin of attraction by $\mathcal{B}(A)$, we may then distinguish between the following two types of riddling: (i) $\mathcal{B}(A)$ is globally riddled if $A$ attracts a positive Lebesgue measure set of points from $U_{\delta}(A)$, but not the full measure. The remaining points, i.e., the points of $U_{\delta}(A)$ not belonging to $\mathcal{B}(A)$, go to some other state. In this case, $A$ is referred to as a weak Milnor attractor [10]. (ii) $\mathcal{B}(A)$ is locally riddled [7] if $A$ attracts almost all points in $U_{\delta}(A)$; i.e., $\operatorname{mes}\left[\mathcal{B}(A) \cap U_{\delta}(A)\right]=\operatorname{mes} U_{\delta}(A)$. $A$ is then said to be a Milnor attractor, and $\mathcal{B}(A)$ contains no observable holes belonging to the basin of another attractor. mes $U_{\delta}(A)$ denotes the Lebesgue measure of $U_{\delta}(A)$.

The purpose of the present Letter is to show how the emergence of locally riddled basins of attraction depends on the existence of a so-called absorbing area $\mathcal{A}(A)$ inside the basin of attraction $\mathcal{B}(A)$. If such an area exists, the nonlinearities of the system restrain trajectories started within $\mathcal{A}(A)$ from coming into contact with the basin boundary. Variation of a parameter that causes the absorbing area to increase in size (or the basin of attraction to shrink) may then lead to a crisis where the 
two boundaries touch one another. After this crisis, the basin of attraction will be globally riddled.

We shall also show that the existence of an absorbing area within the basin of attraction is essential for what happens in the blowout bifurcation, where $\lambda_{\perp}$ becomes positive. In the presence of an absorbing area, the blowout bifurcation will be gradual. The symmetry of the synchronized state will break, and the attractor will spread into the two-dimensional phase space with a more or less pronounced peak of the invariant density near the invariant manifold. This corresponds to a socalled nonhysteric blowout bifurcation [5]. Without the restraining borders of the absorbing area, the blowout bifurcation is similar to an explosion, leading to the abrupt collapse of the synchronized state (hysteric blowout).

Let us consider a system of two coupled logistic maps of the form

$$
F_{a, \varepsilon}:\left\{\begin{array}{l}
x \\
y
\end{array}\right\} \rightarrow\left\{\begin{array}{l}
f_{a}(x)+\varepsilon(y-x) \\
f_{a}(y)+\varepsilon(x-y)
\end{array}\right\},
$$

where $f_{a}: x \rightarrow a x(1-x), x \in R^{1}, a \in[0,4]$ is the one-dimensional logistic map, and $\varepsilon \in[-2,2]$ is the coupling parameter.

In order to delineate the regions in parameter space in which the synchronized state is asymptotically stable, we have determined the regions of transverse stability for the main low-periodic cycles. For an $N$-periodic synchronous state $\gamma_{N}=\left\{x_{1}, x_{2}, \ldots, x_{N}\right\}$, the criterion for transverse stability is

$$
\prod_{n=1}^{N}\left|f_{a}^{\prime}\left(x_{n}\right)-2 \varepsilon\right|<1
$$

Considering, for instance, the fixed point $P\left(x_{0}, x_{0}\right)$ with $x_{0}=1-1 / a$, the condition (2) gives the stability region $-(a-1) / 2<\varepsilon<-(a-3) / 2$. The slanting line denoted fixed point in the middle of Fig. 1 represents the lower edge of this zone. The upper boundary falls outside the range of coupling strengths considered in the figure. Hence, the fixed point is transversely stable to the right of the fixed point line.

The period-2 cycle arises at $a=3$ and hereafter alternates between $x_{1,2}=[a+1 \pm \sqrt{(a+1)(a-3)}] / 2 a$. The condition for transverse stability, in this case, gives two intervals for $\varepsilon$ of which the interval to the left of $\varepsilon=-\frac{1}{2}$ is determined by

$$
\begin{aligned}
-\frac{1}{2}[1+\sqrt{(a+1)(a-3)+1} & <\varepsilon \\
& <-\frac{1}{2}[1+\sqrt{(a+1)(a-3)-1}] .
\end{aligned}
$$

The borderlines of this interval are represented by the curves denoted period 2 in Fig. 1. Similarly, the curves denoted period 4 , period 6 , and period 8 delineate the regions of transverse stability for these cycles. With the values of $a$ considered in the figure, period-3 cycles have not yet appeared in the individual map. Hence, synchronized behavior with this periodicity cannot occur.

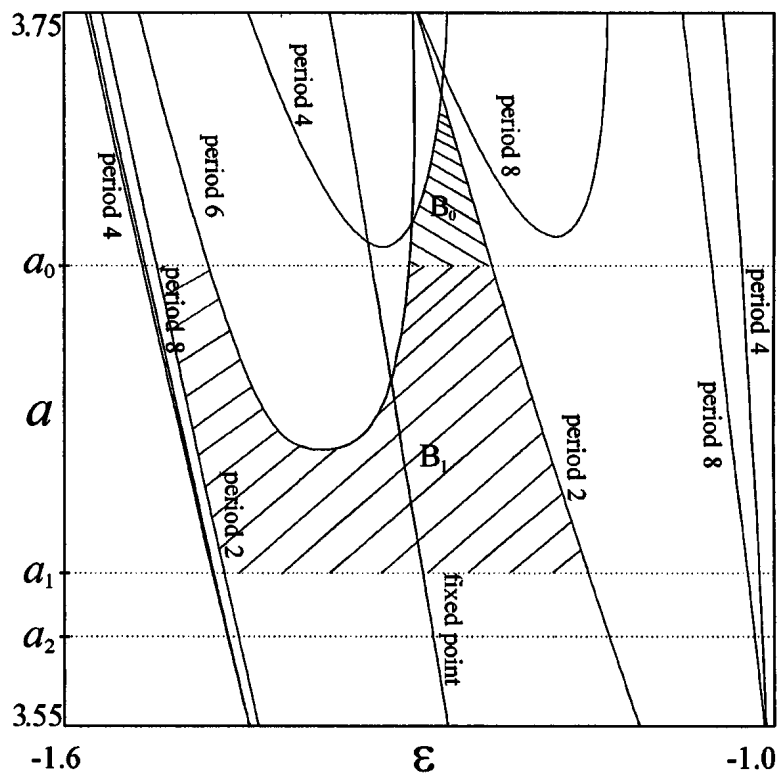

FIG. 1. Riddling bifurcation curves delineating the parameter region of absolute stability.

Cycles of higher periodicity are not likely to be first to become unstable [11].

The logistic map is known to exhibit a positive measure Cantor set of parameter values $a \in[3,4]$ for which a chaotic attractor exists. In particular, this is the case for $a=a_{0}=3.67857351042832 \ldots$, where the fixed point $x_{0}=1-1 / a$ undergoes its first homoclinic bifurcation and for $a=a_{1}=3.59257218410697 \ldots$, where the period-2 cycle $\gamma_{2}=\left\{x_{1}, x_{2}\right\}$ undergoes the first homoclinic bifurcation. At $a=a_{0}, f_{a}(x)$ displays a one-band chaotic attractor $\Gamma=\Gamma_{0}$, which consists of two subbands having contact in $x_{0}$. Similarly, for $a=a_{1}, f_{a}(x)$ produces a two-band chaotic attractor $\Gamma=\Gamma_{1}$, which consists of four subbands having pairwise contact in the period-2 points $x_{1}$ and $x_{2}$.

$\Gamma_{0}$ has an absolutely continuous invariant measure, and almost all trajectories on the synchronized chaotic set $A_{0}=\left\{x=y \in \Gamma_{0}\right\}$ will exhibit the same Lyapunov exponent. Figure 2 shows the variation of the transverse Lyapunov exponent

$$
\lambda_{\perp}=\lim _{N \rightarrow \infty} \frac{1}{N} \sum_{n=1}^{N} \ln \left|f_{a}^{\prime}\left(x_{n}\right)-2 \varepsilon\right|,
$$

with $\varepsilon$ for $a=a_{0}$. Here, $\left\{x_{n}=f_{a}^{n}(x)\right\}_{n=1}^{\infty}$ is a typical itinerary of $f_{a}(x)$. The transverse Lyapunov exponent is negative from $\varepsilon \cong-1.54$ to $\varepsilon \cong-0.48$, and, in this interval, the synchronous state is at least weakly attracting in the Milnor sense [10]. The points where $\lambda_{\perp}$ changes sign are referred to as blowout bifurcations. The region of strong stability is restricted to the interval between $\varepsilon \cong-1.31$, where the period- 6 orbit becomes transversely unstable in a subcritical period-doubling bifurcation, and $\varepsilon \cong-1.24$, where the period- 2 orbit 


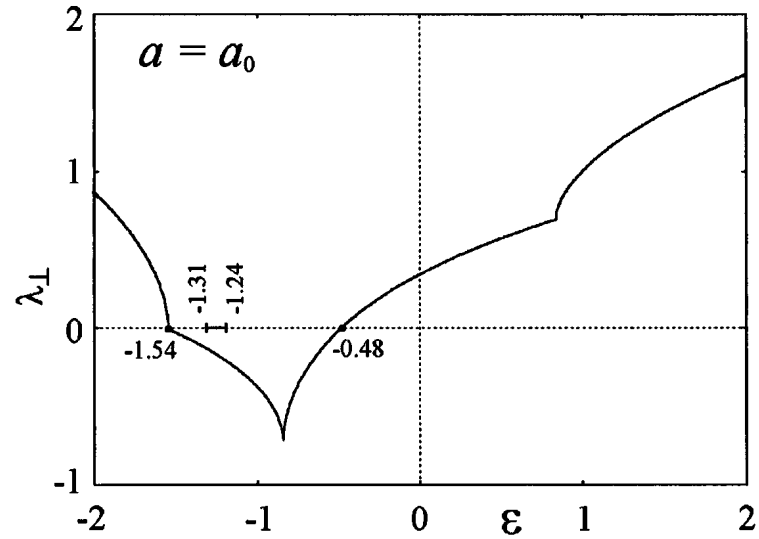

FIG. 2. Transverse Lyapunov exponent as a function of the coupling parameter.

becomes transversely unstable in a supercritical period doubling.

An investigation [9] of the form of the basin of attraction for different values of the coupling parameter shows that to the left of the range of strong stability we have an interval of global riddling with a dense set of repelling tongues leading to divergent orbits. Immediately to the right of this range, on the other hand, while the basin of attraction exhibits a fractal boundary, one cannot observe tongues emanating from the synchronized state and leading the trajectories to diverge. Here, the global dynamics of $F_{a, \varepsilon}$ is such that points that are repelled from the synchronized state never reach the basin boundary. The trajectories are restricted to move within the absorbing area, and this area falls completely within the basin boundary. Hence, we observe the phenomenon of local riddling.

Global analyses of noninvertible maps use the method of critical curves [12]. Similar to the manner in which the iterates of the critical point(s) for a one-dimensional map restrict the regions of phase space in which the stationary trajectory can move, the successive images of the critical curves restrain the dynamics of our two-dimensional map $F_{a, \varepsilon}$. Together, these images define the absorbing area with the properties (i) that once a trajectory has entered this area, it can never leave again, and (ii) all points from the neighborhood will be mapped into the absorbing area in a finite number of iterations.

The critical curves $L_{0}$ are defined as the locus of $|D F|=0$, with $D F$ denoting the Jacobian matrix of $F_{a, \varepsilon}$. For our two-coupled logistic maps, we have

$$
L_{0}=\left\{(x, y) \in R^{2}: y=\frac{2(a-\varepsilon) x-(a-2 \varepsilon)}{4 a x-2(a-\varepsilon)}\right\} .
$$

The boundary $\partial \mathcal{A}$ of the absorbing area may be constructed following the procedure described by Abraham et al. [13]: If $L=\mathcal{A} \cap L_{0}$, then for a suitable integer $m$,

$$
\partial \mathcal{A}=\bigcup_{k=1}^{m} F_{a, \varepsilon}^{k}(L) .
$$

Figure 3 shows the results of such a construction for $a=a_{0}$ and $\varepsilon=-1.234$. Bounded by the curve sections $L_{m}, m=1,2, \ldots, 8$, the absorbing area clearly falls inside the basin boundary $\partial \mathcal{B}$, as represented by the fractal curve. As long as no other attractor exists inside the absorbing area, only local riddling will arise. As $\varepsilon$ is further increased, a crisis occurs (approximately at $\varepsilon=-1.2$ ) in which the border of the absorbing area comes into contact with the basin boundary. Hereafter, the riddling is global until, for $\varepsilon \cong-0.48$, the blowout bifurcation occurs, and the synchronized attractor instantaneously disappears.

When the riddling bifurcation is supercritical, as in the present case, yet another invariant region comes into play. Immediately after the transverse period-doubling at $\varepsilon=-1.237$, almost all trajectories repelled from the synchronized state will be confined to a so-called mixed absorbing area [14] by the unstable manifold of the period-4 saddle cycle. This mixed absorbing area is shown crosshatched in Fig. 3.

For $a=a_{1}$, one can find an interval of $\varepsilon$ in which the order of bifurcations is different. The blowout bifurcation here occurs while an absorbing area still exists inside the basin of attraction. In the whole interval from the riddling bifurcation to the blowout bifurcation, we then observe a locally riddled basin of attraction. At the same time, the blowout bifurcation takes a very different form. Instead of a sudden explosion, the bifurcation manifests itself as a gradual transition from the synchronized chaotic attractor $A_{1}=\left\{x=y \in \Gamma_{1}\right\}$ to an approximately two-dimensional attractor $A_{1}^{\prime}$, which is bounded by the absorbing area. This is demonstrated in Fig. 4 for $\varepsilon=-1.03$.

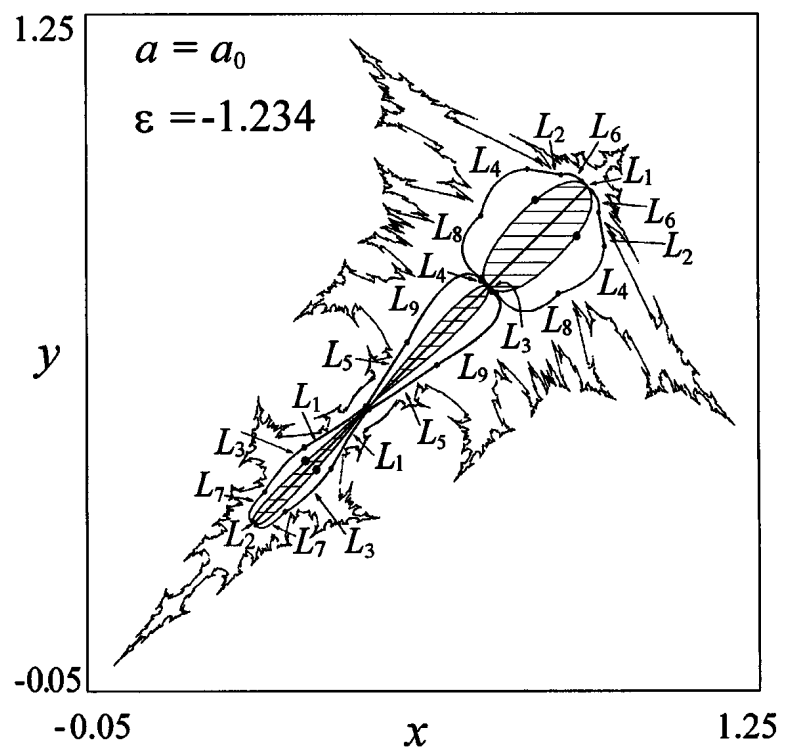

FIG. 3. The absorbing area falls inside the basin boundary. Note also the so-called mixed absorbing area inside $\mathcal{A}(A)$. 


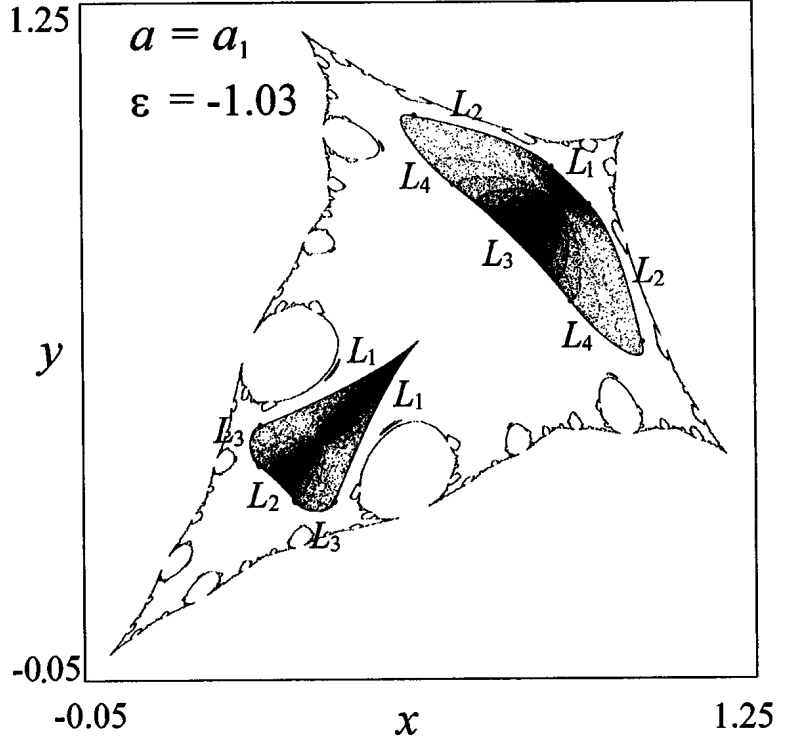

FIG. 4. The blowout bifurcation (at $\varepsilon \cong-1.04$ ) leads to an attractor in two-dimensional phase space.

Just after the blowout, the probability for the system to move in the neighborhood of the synchronized state is large. From time to time, significant bursts may be observed in which the trajectory moves far out in phase space without, however, coming into contact with the basin boundary. This is a typical example of on-off intermittency [3].

We conclude that, besides the riddling bifurcation (at $\varepsilon^{(a)}$ ) and the blowout bifurcation (at $\varepsilon^{(b)}$ ), the contact bifurcation (or crisis at $\varepsilon^{(c)}$ ) in which the boundary of the absorbing area touches the basin boundary is important in accounting for the phenomena observed in connection with the breakdown of chaotic synchronization. If $\varepsilon^{(c)}$ occurs before $\varepsilon^{(b)}$, the system will display local riddling between $\varepsilon^{(a)}$ and $\varepsilon^{(c)}$, and global riddling between $\varepsilon^{(c)}$ and $\varepsilon^{(b)}$. In this case, the blowout bifurcation takes the form of an abrupt explosion. On the other hand, if $\varepsilon^{(c)}$ occurs after $\varepsilon^{(b)}$, there is no global riddling, and the blowout bifurcation is gradual.
A more complicated scenario can arise if other attracting states exist inside the absorbing area [9]. The problem of the existence of such attractors is extremely complicated from the view of the mathematical theory of dynamical systems. It is associated with the possible existence of parameter intervals with nonremovable homoclinic tangencies [15].

We thank T. Kapitaniak, L. Gardini, G.-I. Bischi, and C. Mira for a number of illuminating discussions. J. Laugesen is acknowledged for assistance with the numerical computations.

[1] H. Fujisaka and T. Yamada, Prog. Theor. Phys. 69, 32 (1983).

[2] N. F. Rulkov, Chaos 6, 262 (1996).

[3] N. Platt, E. A. Spiegel, and C. Tresser, Phys. Rev. Lett. 70, 279 (1993).

[4] J. C. Alexander, J. A. Yorke, Z. You, and I. Kan, Int. J. Bifurcation Chaos Appl. Sci. Eng. 2, 795 (1992).

[5] J. C. Sommerer and E. Ott, Nature (London) 365, 136 (1993); E. Ott and J. C. Sommerer, Phys. Lett. A 188, 39 (1994).

[6] J.F. Heagy, T. L. Carroll, and L. M. Pecora, Phys. Rev. Lett. 73, 3528 (1994).

[7] P. Ashwin, J. Buescu, and I. Stewart, Nonlinearity 9, 703 (1996).

[8] Y.-C. Lai, C. Grebogi, J. A. Yorke, and S. C. Venkataramani, Phys. Rev. Lett. 77, 55 (1996).

[9] Yu. L. Maistrenko, V.L. Maistrenko, A. Popovich, and E. Mosekilde (to be published).

[10] J. Milnor, Commun. Math. Phys. 99, 177 (1985).

[11] B. R. Hunt and E. Ott, Phys. Rev. Lett. 76, 2254 (1996).

[12] C. Mira, L. Gardini, A. Barugola, and J.-C. Cathala, Chaotic Dynamics in Two-Dimensional Noninvertible Maps (World Scientific, Singapore, 1996).

[13] R. H. Abraham, L. Gardini, and C. Mira, Chaos in Discrete Dynamical Systems (Springer-Verlag, Berlin, 1997).

[14] C. Mira, C. Rauzy, Yu. Maistrenko, and I. Sushko, Int. J. Bifurcation Chaos Appl. Sci. Eng. 6, 2299 (1996).

[15] S. V. Gonchenko, L.P. Shil'nikov, and D. V. Turaev, Chaos 6, 15 (1996). 